\title{
Treatment-related severe and fatal adverse events with molecular targeted agents in the treatment of advanced gastric cancer: a meta-analysis
}

This article was published in the following Dove Press journal:

OncoTargets and Therapy

26 April 2017

Number of times this article has been viewed

\section{Liang Wang \\ Yagang Liu \\ Wenyong Zhou \\ Wei Li}

Department of General Surgery, The Central Cangzhou Hospital, Cangzhou, Hebei Province, People's Republic of China
Correspondence: Liang Wang Department of General Surgery, The Central Cangzhou Hospital, Cangzhou, Hebei Province 061000, People's

Republic of China

Tel +8603।72075646

Fax +8603I7 2075647

Email wangliang20I60I@sina.com
Aim: To perform a systematic review and meta-analysis of Phase III randomized controlled trials (RCTs) to determine the incidence and risk of severe adverse events (AEs) with molecular targeted agents (MTAs) in advanced/metastatic gastric cancer (GC) patients.

Methods: A comprehensive literature search for related trials published up to December 2015 was performed. Eligible studies were Phase III RCTs of advanced/metastatic GC patients assigned to MTAs or control group. Data were extracted by two authors for severe and fatal AEs (FAEs).

Results: A total of nine Phase III RCTs involved 4,934 GC patients were ultimately identified. The pooled results demonstrated that the addition of TAs to therapies in advanced GC significantly increased the risk of developing severe AEs (relative risk: $1.12,95 \%$ confidence interval: $1.02-1.24, P=0.02$ ), but not for FAEs (relative risk: $0.97,95 \%$ confidence interval: $0.65-1.45$, $P=0.88)$. Additionally, the most common causes of FAEs with MTAs were infections $(16.3 \%)$, gastrointestinal hemorrhage (8.2\%), and arterial thromboembolic events $(8.2 \%)$, respectively. Conclusion: With available evidence, the use of TAs in GC patients was associated with an increased risk of severe AEs, but not for FAE. Clinicians should be aware of the risk of severe AEs with the administration of these drugs in these patients.

Keywords: advanced gastric cancer, molecular targeted agents, randomized, meta-analysis

\section{Introduction}

Gastric cancer (GC) is one of the most common malignant diseases worldwide, accounting for $8 \%(989,600$ million $)$ of the total new cancer cases and $10 \%(738,000)$ of the total cancer deaths in 2008. ${ }^{1}$ Generally, GC is a heterogeneous disease, which usually includes different subgroups according to histological, anatomical, genomic, or molecular classifications. ${ }^{2,3}$ Regardless of these subtypes, the current treatment of GC is based on a multidisciplinary approach that combines gastrectomy, radiotherapy, and chemotherapy. ${ }^{4}$ Despite the advances in the treatment, nearly $50 \%$ of patients with locally advanced-stage GC relapse after gastrectomy. ${ }^{5,6}$ For such patients, palliative chemotherapy is the mainstay treatment to prolong the survival. Currently, combination chemotherapy based on 5-fluoropyrimidines/platinum, with possible addition of docetaxel in fit patients, represent the landmark of first-line treatment of advanced GC patients. However, the efficacy of first-line chemotherapy is modest, with a median survival 8-12 months, ${ }^{7-9}$ and most patients are nonresponders or eventually experience disease progression. Thus, it is clear that novel treatments are badly needed in advanced GC patients. 
During the past decades, a better understanding of the molecular events involved in the tumorigenesis of GCs has led to development of new targeted agents. ${ }^{10}$ A recent meta-analysis conducted by Qi et al ${ }^{11}$ demonstrated that the use of anti-vascular endothelial growth factor (anti-VEGF) agents provided a significant survival benefit in previously treated GC patients. Another meta-analysis conducted by Ciliberto et $\mathrm{al}^{12}$ also showed that antiangiogenic agents (hazard ratio [HR]: 0.759; 95\% confidence interval [CI]: $0.655-0.880 ; P<0.001)$ and anti-HER-2 agents (HR: 0.823; 95\% CI: $0.722-0.939 ; P=0.004)$ significantly improve overall survival, while no benefit was found for anti-EGFR agents (HR: $1.077 ; 95 \%$ CI: $0.847-1.370 ; P=0.543$ ). To date, two molecular targeted agents (MTAs) targeting VEGF signal pathway, bevacizumab and ramucirumab, ${ }^{13}$ and one MTAtargeting EGFR signal pathway, trastuzumab, ${ }^{14}$ have been approved for use in advanced GC patients due to survival benefits. Therefore, it is anticipated that the use of these MTAs in GC would increase in the future. However, VEGF and EGFR play multiple roles in physiologic processes, and thus their inhibition could have potentially serious systemic consequences. To our best knowledge, there is no specific meta-analysis to assess the severe adverse events (AEs) and fatal adverse events (FAEs) associated with MTAs in GC. We, therefore, conduct this comprehensive meta-analysis of Phase III randomized controlled trials (RCTs) to assess the toxicities of MTAs in advanced GC patents.

\section{Materials and methods}

\section{Study design}

We performed this meta-analysis according to the Preferred Reporting Items for Systematic Reviews and Meta-Analyses statements. ${ }^{15}$

\section{Search strategy}

In December 2015, an extensive search of the following databases was performed: Embase, Medline, the Cochrane Central Register of Controlled Trials, and the Cochrane Database of Systematic Reviews. The following keywords were used: "gastric neoplasms," "gastric cancer," "gastric carcinoma," "sorafenib," “unitinib," "pazopanib," "axitinib," "cediranib," "regorafenib," "ramucirumab," "vandetanib," "bevacizumab," "angiogenesis inhibitor," "mTOR inhibitor," "everolimus," "cetuximab," "panitumumab," "lapatinib," "trastuzumab," "molecular targeted agents," and "randomized controlled trials." The language of publication and years were not limited.

\section{Selection of trials}

Clinical trials that met the following criteria were included: 1) Phase III RCTs in patients with pathologically confirmed GC; 2) participants assigned to treatment with or without MTAs; and 3) reported outcomes of interest (ie, severe $\mathrm{AE}$ and FAEs). We used the five-item Jadad scale including randomization, double-blinding, and withdrawals as previously described to approximately assess the quality of included trials. ${ }^{16}$

\section{Data extraction}

Two investigators independently performed data extraction. If reviewers suspected an overlap of cohorts in a report, they contacted the corresponding author for clarification; we excluded studies with a clear overlap. The following information was recorded for each study: first authors' name, year of publication, study period, median age, MTAs dosage, number of patients enrolled, and events of severe and FAEs. The primary end point of this study was FAE, which was defined by the National Cancer Institute's Common Terminology Criteria for Adverse Events as deaths occurring during a clinical trial as a result of exposure to an experimental drug. We did not include FAEs that were related to disease progression. The second end point of this study was severe AE, which was defined by the National Cancer Institute's Common Terminology Criteria for Adverse Events as Grade 3 or 4 toxicities occurring during a clinical trial as a result of exposure to an experimental drug.

\section{Statistical analysis}

Statistical analysis of severe and fatal AEs was calculated using comprehensive meta-analysis software version 2.0 (Biostat, Englewood, NJ, USA). Between-study heterogeneity was estimated using the $\chi^{2}$-based $Q$ statistic. ${ }^{17}$ Heterogeneity was considered statistically significant when $P_{\text {heterogeneity }}<0.05$ or $I^{2}>50 \%$. We calculated the pooled relative risk (RR) and $95 \%$ CIs by using random-effect or fixed-effect models according to the heterogeneity of included studies. A statistical test with a $P$-value less than 0.05 was considered significant. RR $>1$ indicates more toxicities in MTAs group, and vice versa. Finally, publication bias was evaluated through funnel plots and with Begg and Egger's tests. ${ }^{18,19}$

\section{Results \\ Search results}

Our literature search revealed 252 potential relevant publications, and 21 reports were retrieved for full-text evaluation; 


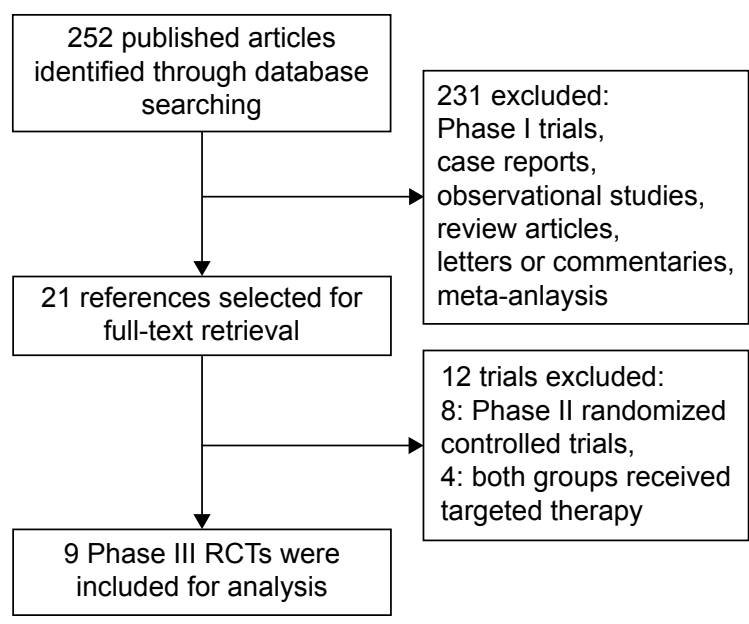

Figure I Studies eligible for inclusion in the meta-analysis. Abbreviation: RCTs, randomized controlled trials.

the reasons for study exclusion are illustrated in Figure 1. Finally, a total of nine RCTs with 4,934 patients were included. ${ }^{20-28}$ The baseline characteristics of each trial are listed in Table 1. Five trials were performed in first-line settings and four in second-line settings (Table 1). ${ }^{20,22,23,25}$ The quality of each included study was approximately assessed according to Jadad scale; five trials were placebo-controlled, double-blinded randomized trials, and thus had Jadad score of 5..$^{20,21,23-28}$ The other four trials had Jadad score of 3.
Incidence of severe and fatal AEs

A total of 2,647 patients from nine treatment arms receiving MTAs were available for severe AEs incidence analysis. Using a random-effects model, the summary incidence of severe AEs was 72.5\% (95\% CI: 66.4\%-77.8\%). As for FAEs, a total of 2,647 patients from nine treatment arms were included, and the pooled incidence was $2.2 \%$ (95\% CI: $1.6 \%-2.9 \%)$ using a fixed-effects model $\left(I^{2}=48 \%, P=0.051\right)$.

\section{RR of severe and fatal AEs}

A meta-analysis of RR for severe and fatal AEs attributable to MTAs compared with control was performed. The pooled results showed that the use of MTAs significantly increased the risk of severe AEs (RR: 1.12, 95\% CI: 1.02-1.24, $P=0.02$; Figure 2), but not FAEs when compared with controls (RR: 0.97, 95\% CI: 0.65-1.45, $P=0.88$; Figure 3) using a fixedeffects model.

\section{Specific FAEs}

Individual specified and nonspecified causes of FAEs are listed in Table 2. There were 49 FAEs on the treatment arms and 46 FAEs on the controlled arms; $42.9 \%$ and $50 \%$ of them were nonspecified etiology, respectively. For those specified FAEs in this study, the most common causes of FAEs with MTAs

Table I Baseline characteristics of nine included trials

\begin{tabular}{|c|c|c|c|c|c|c|c|c|}
\hline Study & $\begin{array}{l}\text { Treatment } \\
\text { line }\end{array}$ & $\begin{array}{l}\text { Total } \\
(\mathbf{N})\end{array}$ & Treatment arms & $\begin{array}{l}\text { Number } \\
\text { for analysis }\end{array}$ & $\begin{array}{l}\text { Median } \\
\text { age (years) }\end{array}$ & $\begin{array}{l}\text { Median } \\
\text { PFS }\end{array}$ & $\begin{array}{l}\text { Median } \\
\text { OS }\end{array}$ & $\begin{array}{l}\text { Jadad } \\
\text { score }\end{array}$ \\
\hline Fuchs et $\mathrm{al}^{23}$ & Second-line & 335 & Ramucirumab 8 mg/kg & 236 & 60 & NR & 5.2 & 5 \\
\hline$(20 \mid 4)$ & & & Placebo & 115 & 60 & NR & 3.8 & \\
\hline Wilke et $\mathrm{al}^{20}$ & First-line & 655 & Ramucirumab 8 mg/kg + PTX & 327 & 61 & 4.4 & 9.6 & 5 \\
\hline$(20 \mid 4)$ & & & Placebo + PTX & 329 & 61 & 2.9 & 7.4 & \\
\hline \multirow[t]{2}{*}{$\begin{array}{l}\text { Shen et } \mathrm{al}^{2 \mathrm{I}} \\
(2015)\end{array}$} & Second-line & 202 & $\begin{array}{l}\text { Bevacizumab } 2.5 \mathrm{mg} / \mathrm{kg} / \mathrm{wk}+ \\
\text { capecitabine + DDP }\end{array}$ & 100 & 54.2 & 6 & II.4 & 5 \\
\hline & & & Placebo + capecitabine + DDP & 102 & 55.5 & 6.3 & 10.5 & \\
\hline Satoh et $\mathrm{al}^{22}$ & Second-line & 261 & Lapatinib I,500 mg qd + PTX & 132 & 61 & 5.4 & II & 3 \\
\hline$(20 \mid 4)$ & & & PTX & 129 & 62 & 4.4 & 8.9 & \\
\hline Waddell et $\mathrm{al}^{24}$ & First-line & 553 & Panitumumab + EOC & 278 & 63 & 7.4 & II.3 & 3 \\
\hline$(2013)$ & & & EOC & 275 & 62 & 6 & 8.8 & \\
\hline Ohtsu et $\mathrm{al}^{25}$ & Second-line & 656 & Everolimus $10 \mathrm{mg} / \mathrm{d}$ & 439 & 62 & 1.7 & 5.4 & 5 \\
\hline$(2013)$ & & & Placebo & 217 & 62 & 1.4 & 4.3 & \\
\hline Lordick et $\mathrm{al}^{26}$ & First-line & 904 & Cetuximab + capecitabine + DDP & 455 & 60 & 4.4 & 9.4 & 3 \\
\hline$(20 \mid 3)$ & & & Capecitabine + DDP & 449 & 59 & 5.6 & 10.7 & \\
\hline \multirow[t]{2}{*}{$\begin{array}{l}\text { Ohtsu et } \mathrm{al}^{27} \\
(20 \mathrm{II})\end{array}$} & First-line & 774 & $\begin{array}{l}\text { Bevacizumab } 2.5 \mathrm{mg} / \mathrm{kg} / \mathrm{wk}+ \\
\text { capecitabine + DDP }\end{array}$ & 386 & 58 & 6.7 & 12.1 & 5 \\
\hline & & & Placebo + capecitabine + DDP & 381 & 59 & 5.3 & 10.1 & \\
\hline Bang et $\mathrm{al}^{28}$ & First-line & 594 & Trastuzumab + chemotherapy & 294 & 59.4 & 6.7 & 13.8 & 3 \\
\hline$(2010)$ & & & Chemotherapy & 290 & 58.5 & 5.5 & II.I & \\
\hline
\end{tabular}

Abbreviations: PTX, paclitaxel; DDP, cisplatin; EOC, epirubicin plus oxaliplatin plus capecitabine; PFS, progression-free survival; OS, overall survival; NR, not reported. 


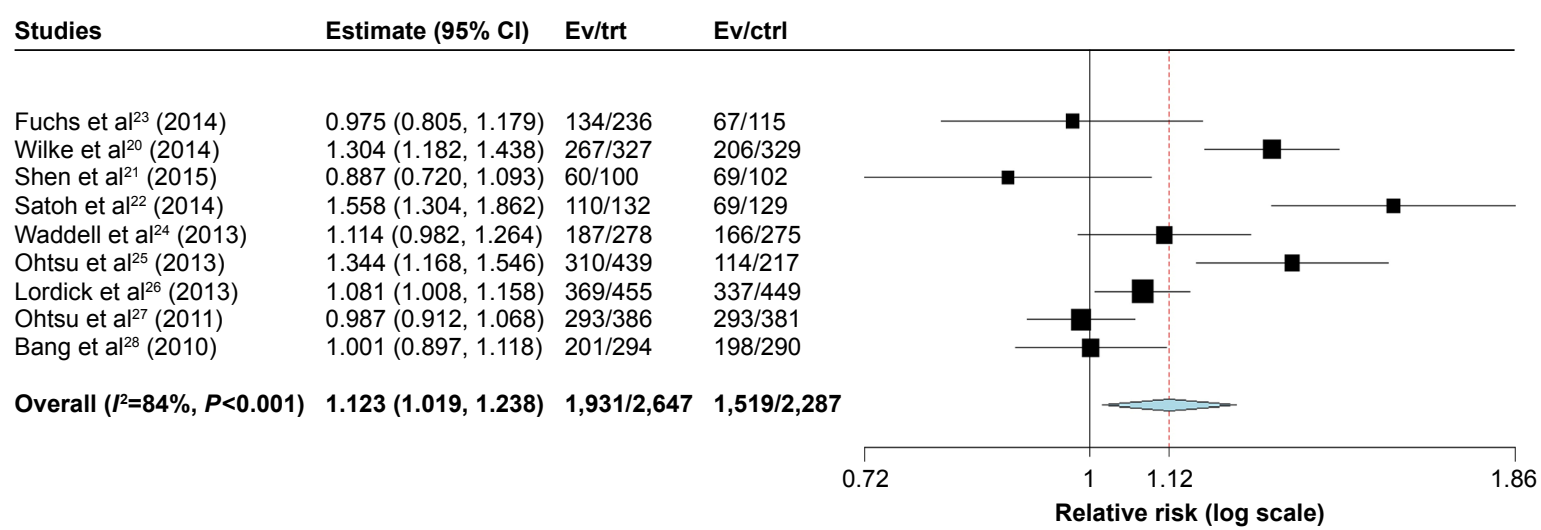

Figure 2 RR of severe AEs $(95 \% \mathrm{Cl})$ associated with therapies with or without MTAs.

Abbreviations: RR, relative risk; AEs, adverse events; MTAs, molecular targeted agents; Cl, confidence interval; Ev, events; trt, treatments; ctrl, control.

were infections $(16.3 \%)$, gastrointestinal hemorrhage $(8.2 \%)$, and arterial thromboembolic events $(8.2 \%)$, respectively. Additionally, the specified FAEs of eight infections were pneumonitis (three), Candida sepsis (one), Klebsiella sepsis (one), sepsis (two), and neutropenic sepsis (one), respectively.

\section{Publication bias}

We used Begg's funnel plot and Egger's test to assess the publication bias. The Begg's funnel plots did not show any evidence of publication bias $(P=0.89$ for severe AEs and $P=0.54$ for FAEs, respectively). Additionally, Egger's test also did not suggest any evidence of publication bias $(P=0.56$ for severe AEs and $P=0.30$ for FAEs, respectively).

\section{Discussion}

During the past decades, the introduction of biological agents targeting specific growth and survival pathways, such as EGFR, PI3K/Akt/mTOR pathway, and angiogenesis through the VEGF signaling cascade, seems to be the most promising strategy to improve outcome of advanced GC patients. Trastuzumab in combination with chemotherapy has been approved by US Food and Drug Administration (FDA) as first-line treatment for patients with HER2-positive advanced gastric or gastroesophageal junction cancer due to its survival benefit when compared to chemotherapy alone. ${ }^{28}$ More recently, ramucirumab, a monoclonal antibody VEGFR-2 antagonist, in combination with paclitaxel also significantly increased overall survival in previously treated patients with advanced GC compared with paclitaxel, which led to its approval for use in second-line treatment for advanced GC. ${ }^{29}$ However, no clear survival benefit was experienced with agents targeting EGFR (cetuximab and panitumumab), VEGF-A (bevacizumab), or mTOR (everolimus). Because of the wide use of MTAs in GC patients, concerns have arisen regarding the risk of severe and fatal AEs with these drugs. Indeed, several previous meta-analyses have been performed to assess the severe and fatal toxicities associated with these MTAs. For example, a previous meta-analysis conducted by Ranpura et $\mathrm{al}^{30}$ showed that the addition of bevacizumab

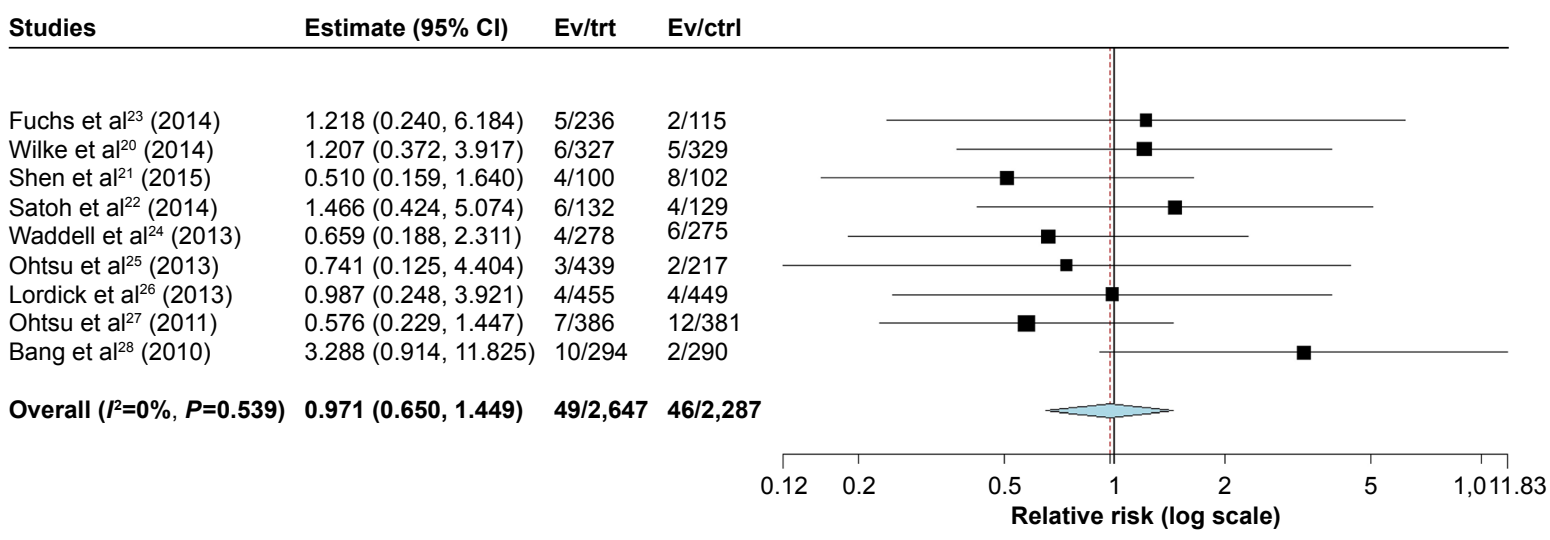

Figure 3 RR of FAEs $(95 \% \mathrm{Cl})$ associated with therapies with or without MTAs.

Abbreviations: RR, relative risk; FAEs, fatal adverse events; MTAs, molecular targeted agents; Cl, confidence interval; Ev, events; trt, treatments; ctrl, control. 
Table 2 FAEs by specific type

\begin{tabular}{lll}
\hline & $\begin{array}{l}\text { Events on } \\
\text { MTAs groups }\end{array}$ & $\begin{array}{l}\text { Events on } \\
\text { control arms }\end{array}$ \\
\hline Unspecified & 21 & 23 \\
Specified & & \\
$\quad$ Infections & 8 & 7 \\
Hemorrhage & 4 & 2 \\
Arterial thromboembolic events & 4 & 2 \\
Pulmonary embolism & 2 & 2 \\
Gl perforation & 2 & $\mathrm{I}$ \\
Sudden death & 3 & 2 \\
Diarrhea & $\mathrm{I}$ & $\mathrm{I}$ \\
Malabsorption & 2 & - \\
Renal failure & - & 2 \\
Cardiac failure & $\mathrm{I}$ & $\mathrm{I}$ \\
Cardiac arrest & $\mathrm{I}$ & - \\
Subileus & - & $\mathrm{I}$ \\
Multiorgan failure & - & $\mathrm{I}$ \\
Dehydration & - & $\mathrm{I}$ \\
Overall & 49 & 46 \\
\hline
\end{tabular}

Abbreviations: FAEs, fatal adverse events; Gl, gastrointestinal; MTAs, molecular targeted agents.

to chemotherapy significantly increased treatment-related mortality (RR: $1.33,95 \%$ CI: $1.02-1.73, P=0.04$ ), and two later meta-analyses also demonstrated that the use of VEGF receptor tyrosine kinase inhibitors was associated with increased risk of FAEs ${ }^{29-32}$ Increased risk of severe and fatal AEs associated with anti-EGFR agent cetuximab has also been observed in colorectal cancer patients. ${ }^{33}$ Additionally, two recent meta-analyses have demonstrated that the use of mTOR inhibitors significantly increase the risk of FAEs. ${ }^{34,35}$ To the best of our knowledge, there is limited data specifically focusing on the severe and fatal AEs related to MTAs in advanced GC patients. Therefore, we conducted this metaanalysis of Phase III RCTs with available toxicity data of MTAs in advanced GC patients.

Our study includes a total of nine Phase III RCTs involving 4,934 GC patients. The summary incidence of severe and fatal AEs with MTAs was $72.5 \%$ and $2.2 \%$, respectively. We also find that the addition of MTAs to therapies in advanced GC significantly increased the risk of developing of severe AEs, but not for FAEs. Additionally, the most common causes of FAEs with MTAs were infections (16.3\%), gastrointestinal hemorrhage (8.2\%), and arterial thromboembolic events $(8.2 \%)$, respectively. On the basis of our findings, we agree with the continued use of MTAs in GC patients owing to its survival benefits, but suggest close monitoring for treatment-related complications. In this study, we also find that infections are the most common FAEs associated with MTAs, which is consistent with previous studies. ${ }^{31-34}$ In a recent meta-analysis conducted by Qi et al, ${ }^{36}$ the authors found that the risk of developing an infection of any grade was 1.45-fold higher in patients treated with bevacizumab. More importantly, there was a 1.59-fold increase in the risk of high-grade infection associated with the use of bevacizumab. Another meta-analysis also demonstrated that the use of antiEGFR MoAbs significantly increased the risk of developing severe infections (RR: $1.34,95 \% \mathrm{CI}: 1.10-1.62, P=0.003$ ) in cancer patients. More importantly, the use of anti-EGFR MoAbs significantly increased the risk of severe sepsis in cancer patients (RR: $4.30,95 \%$ CI: $1.80-10.27, P=0.001$ ). ${ }^{37}$ On the basis of these findings, clinicians should pay more attention to severe infections to reduce the risk of FAEs in advanced gastric patients. Before the initiation of MTAs in gastric patients, clinicians should fully treat patients with any active infection and must monitor patients during the course of MTAs treatment. However, patients with active or recently active infections are excluded from clinical trials; therefore, the true incidence of these infections could be widely underreported. More trials focusing on this issue are still needed.

Several limitations exist in this analysis. First, although AEs are prospectively collected for each individual study, this analysis remains a retrospective research that is subject to the method deficiencies of the included trials. We minimized the likelihood of bias by strictly selecting Phase III RCTs with direct comparison with and without MTAs before the analysis. Second, we included patients treated with different targeted agents, which would increase the clinical heterogeneity among included trials, which also makes the interpretation of a meta-analysis more problematic, although we pooled subgroup analysis according to treatment line. Finally, in the meta-analysis of published studies, publication bias is important because trials with positive results are more likely to be published and trials with null results tend not to be published. Our research detects no publication bias using Begg and Egger tests for severe and fatal AEs.

\section{Conclusion}

In conclusion, this is the first meta-analysis that specifically assessed the severe and fatal toxicities of adding MTAs to therapies in the treatment of $\mathrm{GC}$ patients. The results of our study suggest that the addition of MTAs to therapies in GC significantly increases the risk of developing severe AEs, but not for FAEs. Additionally, the most common causes of FAEs with MTAs were infections, gastrointestinal hemorrhage, and arterial thromboembolic events, respectively.

\section{Disclosure}

The authors report no conflicts of interest in this work. 


\section{References}

1. Jemal A, Bray F, Center MM, Ferlay J, Ward E, Forman D. Global cancer statistics. CA Cancer J Clin. 2011;61(2):69-90.

2. Aoyagi K, Kouhuji K, Kizaki J, Isobe T, Hashimoto K, Shirouzu K. Molecular targeting to treat gastric cancer. World J Gastroenterol. 2014;20(38): 13741-13755.

3. Salimans MM, van Bussel MJ, Brown CS, Spaan WJ. Recombinant parvovirus B19 capsids as a new substrate for detection of B19-specific IgG and IgM antibodies by an enzyme-linked immunosorbent assay. J Virol Methods. 1992;39(3):247-258.

4. Cunningham D, Jost LM, Purkalne G, Oliveira J, Force EGT. ESMO minimum clinical recommendations for diagnosis, treatment and follow-up of gastric cancer. Ann Oncol. 2005;16(Suppl 1):i22-i23.

5. Sasako M, Sakuramoto S, Katai H, et al. Five-year outcomes of a randomized Phase III trial comparing adjuvant chemotherapy with S-1 versus surgery alone in stage II or III gastric cancer. J Clin Oncol. 2011;29(33):4387-4393.

6. Ajani JA. Evolving chemotherapy for advanced gastric cancer. Oncologist. 2005;10(Suppl 3):49-58.

7. Glimelius B, Hoffman K, Haglund U, Nyrén O, Sjödén PO. Initial or delayed chemotherapy with best supportive care in advanced gastric cancer. Ann Oncol. 1994;5(2):189-190.

8. Pyrhönen S, Kuitunen T, Nyandoto P, Kouri M. Randomised comparison of fluorouracil, epidoxorubicin and methotrexate (FEMTX) plus supportive care with supportive care alone in patients with nonresectable gastric cancer. Br J Cancer. 1995;71(3):587-591.

9. Ford HE, Marshall A, Bridgewater JA, et al. Docetaxel versus active symptom control for refractory oesophagogastric adenocarcinoma (COUGAR-02): an open-label, Phase 3 randomised controlled trial. Lancet Oncol. 2014;15(1):78-86.

10. Wong H, Yau T. Targeted therapy in the management of advanced gastric cancer: are we making progress in the era of personalized medicine? Oncologist. 2012;17(3):346-358.

11. Qi WX, Shen Z, Tang LN, Yao Y. The role of anti-VEGF agents in the treatment of advanced gastric cancer: a meta-analysis of randomized controlled trials. Tumour Biol. 2014;35(8):7675-7683.

12. Ciliberto D, Staropoli N, Caglioti F, et al. A systematic review and meta-analysis of randomized trials on the role of targeted therapy in the management of advanced gastric cancer: evidence does not translate? Cancer Biol Ther. 2015;16(8):1148-1159.

13. Abdel-Rahman O. Targeting vascular endothelial growth factor (VEGF) pathway in gastric cancer: preclinical and clinical aspects. Crit Rev Oncol Hematol. 2015;93(1):18-27.

14. Bang YJ. Advances in the management of HER2-positive advanced gastric and gastroesophageal junction cancer. J Clin Gastroenterol. 2012;46(8):637-648.

15. Carrato A, Swieboda-Sadlej A, Staszewska-Skurczynska M, et al. Fluorouracil, leucovorin, and irinotecan plus either sunitinib or placebo in metastatic colorectal cancer: a randomized, Phase III trial. $J$ Clin Oncol. 2013;31(10):1341-1347.

16. Moher D, Pham B, Jones A, et al. Does quality of reports of randomised trials affect estimates of intervention efficacy reported in meta-analyses? Lancet. 1998;352(9128):609-613.

17. Zintzaras E, Ioannidis JP. Heterogeneity testing in meta-analysis of genome searches. Genet Epidemiol. 2005;28(2):123-137.

18. Yusuf S, Peto R, Lewis J, Collins R, Sleight P. Beta blockade during and after myocardial infarction: an overview of the randomized trials. Prog Cardiovasc Dis. 1985;27(5):335-371.

19. Furuse K, Kawahara M, Hasegawa K, et al. Early Phase II study of S-1, a new oral fluoropyrimidine, for advanced non-small-cell lung cancer. Int J Clin Oncol. 2001;6(5):236-241.

20. Wilke H, Muro K, Van Cutsem E, et al. Ramucirumab plus paclitaxel versus placebo plus paclitaxel in patients with previously treated advanced gastric or gastro-oesophageal junction adenocarcinoma (RAINBOW): a double-blind, randomised Phase III trial. Lancet Oncol. 2014;15(11):1224-1235.
21. Shen L, Li J, Xu J, et al. Bevacizumab plus capecitabine and cisplatin in Chinese patients with inoperable locally advanced or metastatic gastric or gastroesophageal junction cancer: randomized, doubleblind, Phase III study (AVATAR study). Gastric cancer. 2015;18(1): 168-176.

22. Satoh T, Xu RH, Chung HC, et al. Lapatinib plus paclitaxel versus paclitaxel alone in the second-line treatment of HER2-amplified advanced gastric cancer in Asian populations: TyTAN - a randomized, Phase III study. J Clin Oncol. 2014;32(19):2039-2049.

23. Fuchs CS, Tomasek J, Yong CJ, et al. Ramucirumab monotherapy for previously treated advanced gastric or gastro-oesophageal junction adenocarcinoma (REGARD): an international, randomised, multicentre, placebo-controlled, Phase III trial. Lancet. 2014;383(9911):31-39.

24. Waddell T, Chau I, Cunningham D, et al. Epirubicin, oxaliplatin, and capecitabine with or without panitumumab for patients with previously untreated advanced oesophagogastric cancer (REAL3): a randomised, open-label Phase III trial. Lancet Oncol. 2013;14(6):481-489.

25. Ohtsu A, Ajani JA, Bai YX, et al. Everolimus for previously treated advanced gastric cancer: results of the randomized, double-blind, Phase III GRANITE-1 study. J Clin Oncol. 2013;31(31):3935-3943.

26. Lordick F, Kang YK, Chung HC, et al. Capecitabine and cisplatin with or without cetuximab for patients with previously untreated advanced gastric cancer (EXPAND): a randomised, open-label Phase III trial. Lancet Oncol. 2013;14(6):490-499.

27. Ohtsu A, Shah MA, Van Cutsem E, et al. Bevacizumab in combination with chemotherapy as first-line therapy in advanced gastric cancer: a randomized, double-blind, placebo-controlled Phase III study. J Clin Oncol. 2011;29(30):3968-3976.

28. Bang YJ, Van Cutsem E, Feyereislova A, et al. Trastuzumab in combination with chemotherapy versus chemotherapy alone for treatment of HER2-positive advanced gastric or gastro-oesophageal junction cancer (ToGA): a Phase III, open-label, randomised controlled trial. Lancet. 2010;376(9742):687-697.

29. Zhang Y, Wu S. Novel therapy for advanced gastric cancer. World $J$ Gastrointest Oncol. 2015;7(11):263-270.

30. Ranpura V, Hapani S, Wu S. Treatment-related mortality with bevacizumab in cancer patients: a meta-analysis. JAMA. 2011;305(5):487-494.

31. Sivendran S, Liu Z, Portas LJ Jr, et al. Treatment-related mortality with vascular endothelial growth factor receptor tyrosine kinase inhibitor therapy in patients with advanced solid tumors: a meta-analysis. Cancer Treat Rev. 2012;38(7):919-925.

32. Schutz FA, Je Y, Richards CJ, Choueiri TK. Meta-analysis of randomized controlled trials for the incidence and risk of treatment-related mortality in patients with cancer treated with vascular endothelial growth factor tyrosine kinase inhibitors. J Clin Oncol. 2012;30(8):871-877.

33. Zhang D, Ye J, Xu T, Xiong B. Treatment related severe and fatal adverse events with cetuximab in colorectal cancer patients: a metaanalysis. J Chemother. 2013;25(3):170-175.

34. Qi WX, Huang YJ, Yao Y, Shen Z, Min DL. Incidence and risk of treatment-related mortality with mTOR inhibitors everolimus and temsirolimus in cancer patients: a meta-analysis. PLoS One. 2013;8(6): e65166.

35. Choueiri TK, Je Y, Sonpavde G, et al. Incidence and risk of treatmentrelated mortality in cancer patients treated with the mammalian target of rapamycin inhibitors. Ann Oncol. 2013;24(8):2092-2097.

36. Qi WX, Fu S, Zhang Q, Guo XM. Bevacizumab increases the risk of infections in cancer patients: a systematic review and pooled analysis of 41 randomized controlled trials. Crit Rev Oncol Hematol. 2015;94(3): 323-336.

37. Qi WX, Fu S, Zhang Q, Guo XM. Incidence and risk of severe infections associated with anti-epidermal growth factor receptor monoclonal antibodies in cancer patients: a systematic review and meta-analysis. BMC Med. 2014;12:203. 
OncoTargets and Therapy

\section{Publish your work in this journal}

OncoTargets and Therapy is an international, peer-reviewed, open access journal focusing on the pathological basis of all cancers, potential targets for therapy and treatment protocols employed to improve the management of cancer patients. The journal also focuses on the impact of management programs and new therapeutic agents and protocols on

patient perspectives such as quality of life, adherence and satisfaction. The manuscript management system is completely online and includes a very quick and fair peer-review system, which is all easy to use. Visit http://www.dovepress.com/testimonials.php to read real quotes from published authors.

Submit your manuscript here: http://www.dovepress.com/oncotargets-and-therapy-journal 